EPJ Web of Conferences 59, 03009 (2013)

DOI: $10.1051 /$ epjconf/20135903009

(C) Owned by the authors, published by EDP Sciences, 2013

\title{
Design of a cone target for fast ignition
}

\author{
Atsushi Sunahara ${ }^{1, a}$, Tomoyuki Johzaki ${ }^{1}$, Hideo Nagatomo², Kunioki Mima² \\ and FIREX project team ${ }^{2}$
}

${ }^{1}$ Institute for Laser Technology

${ }^{2}$ Institute of Laser Engineering, Osaka University

\begin{abstract}
We propose a new type of target for the fast ignition of inertial confinement fusion. Pre-formed plasma inside a cone target can significantly reduce the energy coupling efficiency from the ultra-high intense short-pulse laser to the imploded core plasma. Also, in order to protect the tip of the cone and reduce generation of pre-formed plasma, we propose pointed shaped cone target. In our estimation, the shock traveling time can be delayed $20-30$ ps by lower-Z material with larger areal density compared to the conventional gold flat tip. Also, the jet flow can sweep the blow-off plasma from the tip of the cone, and the implosion performance is not drastically affected by the existence of pointed tip. In addition, the selfgenerated magnetic field is generated along the boundary of cone tip and surrounding CD or DT plasma. This magnetic field can confine fast electrons and focus to the implosion core plasma. Resultant heating efficiency is improved by $30 \%$ compared to that with conventional gold flat tip.
\end{abstract}

\section{INTRODUCTION}

In order to achieve a high-gain of the thermonuclear burn in the fast-ignition scheme of inertial confinement fusion, we need to increase the energy coupling efficiency between the ultra-high intense short-pulse laser and the compressed core. Kodama et al. [1] conducted the first experiment on this fast ignition in 2000. They observed an increased neutron yield from the highly dense core plasma heated by the ultra-high intense laser. Also, they showed the neutron yield scalability with the heating laser energy [2]. They achieved the large energy coupling ranging from $15 \%$ to $30 \%$ between the ultraintense laser and the imploded plasma core. Then, Osaka University started the fast ignition realization experiment (FIREX) [3]. In the 2010 FIREX experiment, we had neutron yields more than that observed by Kodama [2]. However, the energy coupling efficiency between ultra intense laser and the imploded core is estimated to be $10 \%$ [4], still small. We conjecture that the primary reason of small coupling efficiency is the presence of pre-formed plasma inside the cone target. Baton [5] pointed out that preformed plasma inside the cone significantly disturbs the flow of fast electron to the core. Cai [6] showed that existence of the pre-formed plasma inside the cone reduces creation of fast electrons having the energy less than $5 \mathrm{MeV}$. These electrons are the dominant contributor to heating of the core plasma. Johzaki et al. [7] simulated generation of fast electrons using ultra-high intense laser and its transport to the core plasma, and they estimated that $10 \mu \mathrm{m}$ scale pre-formed plasma lowered the coupling efficiency by $80 \%$, compared to the case of $1 \mu \mathrm{m}$. In order to improve the energy coupling efficiency, understanding of pre-formed plasma and its level is important. Therefore, by simulation, we estimated the current level of the pre-plasma formation. Also, in order to relax the shock impact from the imploded plasma on the tip of the cone target, and decreased the pre-formed plasma caused by the shock break-through, we

\footnotetext{
ae-mail: suna@ile.osaka-u.ac.jp
}

This is an Open Access article distributed under the terms of the Creative Commons Attribution License 2.0, which permits unrestricted use, distribution, and reproduction in any medium, provided the original work is properly cited. 
propose a new type of the cone target "TONGARI" (pointed) tip. This pointed tip has another advantage of focusing fast electrons to the core plasma, and is expected to improve the heating energy coupling efficiency.

\section{PRE-PLASMA LEVEL OF FIREX}

We simulated pre-plasma formation inside the cone, using the pre-pulse estimated in FIREX 2009 [8]. The laser wavelength is set to $1.06 \mu \mathrm{m}$. The spot diameter is $90 \mu \mathrm{m}$ (Gaussian FWHM). In the experiment, the measured spot diameter of the ultra-intense short pulse laser was 40-90 $\mu \mathrm{m}$. We assume the spot diameter of the pre-pulse to be the same as that of ultra-intense laser. The laser temporal profile is a flat top. The diameter of the tip of the cone target is $30 \mu \mathrm{m}$, and the opening angle of the cone was set to $45 \mathrm{deg}$. The laser intensities are set to be $10^{12} \mathrm{~W} / \mathrm{cm}^{2}$ and $10^{13} \mathrm{~W} / \mathrm{cm}^{2}$, respectively. The wall thicknesses of the cone tip and the wall near the tip are both $10 \mu \mathrm{m}$. In the simulation, we use tin for the target cone material, instead of gold used in the experiment. This is because we have an accurate tin opacity table, verified by the research of extreme ultra-violet light source [9]. In our simulation, we used two-dimensional radiation hydro code STAR2D [10] with the Euler cylindrical coordinates. Magnetic field effect is not taken into account in line. In Figs. 1(a) and 1(b), we show the profiles of ion and electron densities, and electron temperature after $1 \mathrm{~ns}$ duration pre-pulse irradiation of the intensity of (a) $10^{12} \mathrm{~W} / \mathrm{cm}^{2}$, and (b) $10^{13} \mathrm{~W} / \mathrm{cm}^{2}$, respectively. In Fig. 1(a), we see that a long scale pre-plasma is formed around the critical density of $10^{21} \mathrm{~cm}^{-3}$ for $1.06 \mu \mathrm{m}$ laser, whose scale length $\left(n_{e} / \nabla n_{e}\right)=13 \mu \mathrm{m}$ between the electron number density $n_{e}=10^{21} \mathrm{~cm}^{-3}$ and $n_{e}=3 \times 10^{20} \mathrm{~cm}^{-3}$. Also, below the density of $n_{e}=3 \times 10^{20} \mathrm{~cm}^{-3}$, we see formation of a very long scale pre-plasma. This pre-plasma is mainly created by the ablated plasma from the side walls. Ablated plasma flows collisionally along the axis of the cone. The position of the critical density was shifted only by $10 \mu \mathrm{m}$. The ionization degree at the critical density is 7 . In Fig. 1(b), we show the case of the intensity of $10^{13} \mathrm{~W} / \mathrm{cm}^{2}$. In this case, we see a longer pre-plasma compared to that for $10^{12} \mathrm{~W} / \mathrm{cm}^{2}$. The density scale length between $n_{e}=10^{21} \mathrm{~cm}^{-3}$ and $n_{e}=3 \times 10^{20} \mathrm{~cm}^{-3}$ is $40 \mu \mathrm{m}$. However, the density scale length over the critical density is shorter than that for $10^{12} \mathrm{~W} / \mathrm{cm}^{2}$. This is because the higher ionization degree of 22 resulted from heating by the higher intensity pulse. Comparing Figs. 1(a) and 1(b), at the higher intensity, we see relatively shorter scale length over the critical density and longer scale length below the critical density than at the lower intensity. In the actual experimental condition, there is a pedestal component of the pulse whose intensity increases exponentially with time just before the main pulse. However, this is not taken into account in the present simulation. This pedestal component was not measured in FIREX 2009 experiments, but it exists. It may change the temperature profile, and ionization level in particular. The effect of the pedestal component will be investigated in the future. From Particle in Cell simulations, significant reduction of the energy coupling efficiency is seen from comparison of the pre-plasma scales of $1 \mu \mathrm{m}$ and $10 \mu \mathrm{m}$ [11]. Since we intend to reduce the pre-pulse and the resulted pre-plasma scale to the order of $1 \mu \mathrm{m}$ in future FIREX experiments, understanding of pre-plasma is important. Another cause of the pre-plasma generation inside the cone target is the shock break-though from the imploded core plasma. To suppress this, we propose a new type of cone target, "TONGARI (pointed)" target as described in the next section.

\section{TONGARI TIP}

Protection of the cone tip is very important in future design, since the implosion energy increases to the ignition and thermonuclear burn in the future, high-pressure shock waves can easily destroy the cone tip. To prevent this, we propose use of a pointed (TONGARI) cone tip shown in Fig. 2. The pointed shape can increase the shock-traveling time between the imploded core and inner surface of the cone tip. We simulated the shock propagation for aluminum TONGARI tip from the implosion plasma in the typical implosion condition of FIREX. The length from the inner surface to the tip is $50 \mu \mathrm{m}$, and the tip 
(a)

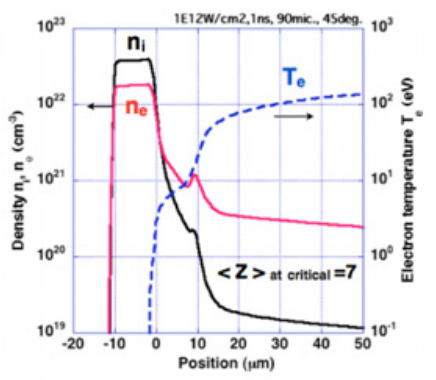

(b)
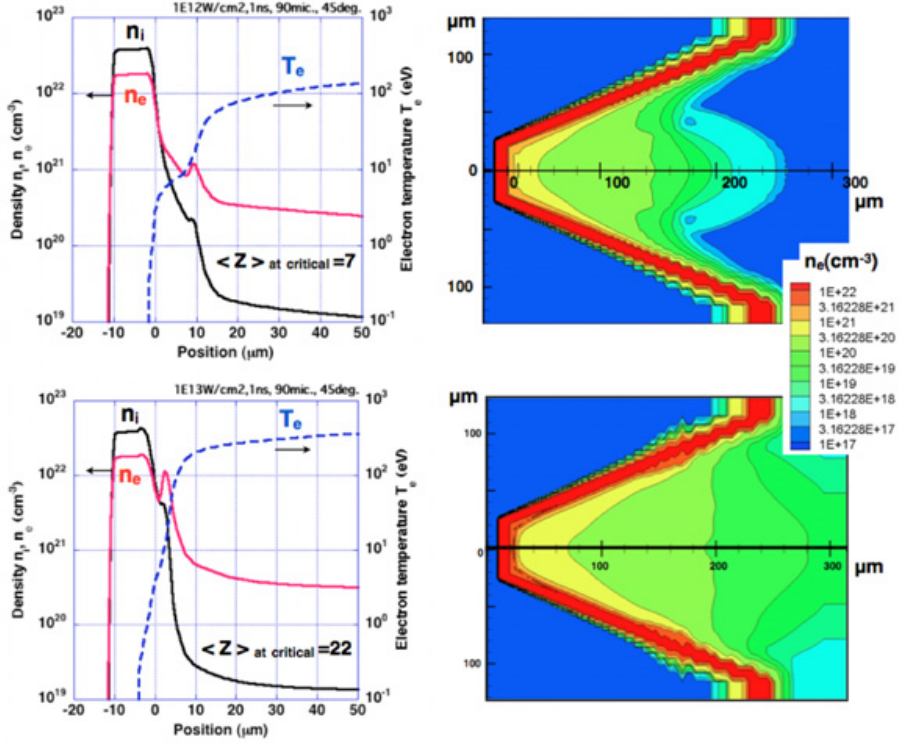

Figure 1. (a) Ion and electron densities after $1 \mathrm{~ns}$ pre-pulse irradiation with $10^{12} \mathrm{~W} / \mathrm{cm}^{2}$ of pre-pluse, (b) $10^{13} \mathrm{~W} / \mathrm{cm}^{2}$ of pre-pulse.

is located at the center of the implosion. The areal density $\rho \Delta \ell$ is $0.0135 \mathrm{~g} / \mathrm{cm}^{2}$ for the aluminum of the solid density $2.7 \mathrm{~g} / \mathrm{cm}^{3}$ and tip length of $50 \mu \mathrm{m}$. Even though this is smaller than $\rho \Delta \ell=0.0193 \mathrm{~g} / \mathrm{cm}^{2}$ of the value for the conventional $10 \mu \mathrm{m}$ thickness gold flat tip, we can increase the delay time to 2030 ps. From the fast electron transport point of view, the low-Z material is better for the tip material, because of less scattering compared to high-Z material such as gold. We use diamond-like carbon (DLC) or aluminum of the solid density 2 to $3 \mathrm{~g} / \mathrm{cm}^{3}$.

In our estimation, tip of TONGARI is set to be center of the shell target. On the other hand, the gap length between the gold flat tip and center of the shell target is $50 \mu \mathrm{m}$. However, in our simulation, the jet flow hitting the tip of TONGARI can sweep the blow-off plasma from the tip of TONGARI. Resultantly, the implosion performance is not drastically affected by the existence of pointed tip. Mixing of the fuel with the tip blow-off plasma is not observed clearly in our simulation. However sub-cell scale mixing may occur, and should be examined in a future work. Also, TONGARI cone tip has an advantage for focusing fast electrons. We show the density and temperature profiles of TONGARI tip at 2.63 ns before the impact in Fig. 2(a), and at $2.77 \mathrm{~ns}$ after the impact in Fig. 2(b) for typical FIREX implosion condition. In Fig. 2(b), the tip of the cone is not damaged so much after the shock impact, and the pointed shape is still maintained. Also the tip after the impact is surrounded by the high temperature implosion plasma. The plasma resistivity is given as a function of the degree of ionization and the temperature [12]. At this time after the impact, the resistivity increases from the implosion plasma of the low density and high temperature to the pointed tip of the high density and low temperature. The resistivity of plasma of DLC or aluminum pointed tip is higher than that of the plasma surrounding CH or DT plasma. When fast electrons flow in this resistive plasma having the gradient of resistivity, a magnetic field $B_{\theta}$ will be generated along the boundary between the implosion plasma and the pointed tip [13];

$$
\frac{\partial B_{\theta}}{\partial t} \propto \nabla \times\left(\eta j_{f}\right)
$$

here, $\eta$ is the resistivity and $j_{f}$ is the fast electron current. Subsequently the magnetic field will focus the electron flow to the imploded core. Most part of TONGARI tip is heated up to and beyond $10 \mathrm{eV}$ 
(a)

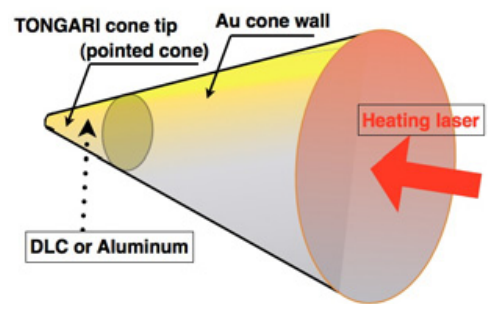

Figure 2. Cone target, "TONGARI".

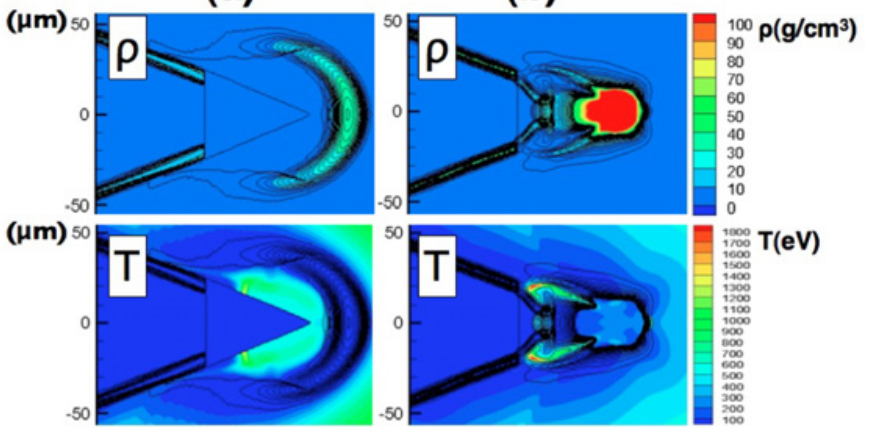

Figure 3. The density and temperature profile at (a) $2.63 \mathrm{~ns}$ before the shock impact, and (b) $2.77 \mathrm{~ns}$ (maximum compression timing).

by the propagating shock from the implosion at the timing of irradiation of the main heating pulse. This justifies the usage of Spitzer resistivity in evaluating the B-field. Using this DLC TONGARI tip, we estimated the growth of the magnetic field, and focusing fast electrons by PIC and Fokker-Planck simulations [14]. Generated magnetic field is up to $10^{3}$ Tesla, and the energy coupling between heating laser and core plasma is higher than that with the conventional gold tip with $5 \mu$ m thickness about $30 \%$. Resultant temperature of the core is $90 \%$ improved at the center.

\section{CONCLUSION}

From 2D radiation simulations, we estimated the pre-pulse level to be 65 to $658 \mathrm{~mJ}$, using $90 \mu \mathrm{m}$ laser spot. The estimated density gradient scale length of the pre-formed plasma inside the cone is 13$40 \mu \mathrm{m}$ between the critical density of $1.06 \mu \mathrm{m}$ laser wavelength and $1 / 3$ of it in the FIREX experiment. This density scale length of pre-formed plasma is still too large for achieving an efficient fast electron production from the heating point of view. In order to reduce pre-formed plasma, we proposed use of TONGARI pointed cone to prevent the damage of the cone tip, before irradiation of the main heating pulse, from the break through of the shock wave from the implosion plasma. Our simulation analysis shows that an aluminum pointed tip delays the shock arrival time by $20 \mathrm{ps,} \mathrm{compared} \mathrm{to} \mathrm{the} \mathrm{conventional}$ gold tip of $10 \mu \mathrm{m}$ used in the typical implosion of GXII laser. Even though the tip of TONGARI is set at the center of the shell target, the implosion performance is not drastically affected by the existence of pointed tip in our simulation. Also we considered the growth of the magnetic field around the tip. Its magnetic field is estimated to be $10^{3}$ Tesla and magnetic field focus fast electrons to the imploded plasma core. The heating energy coupling is improved by $30 \%$, and the temperature at the center of the core plasma is $90 \%$ higher than that for the gold $5 \mu \mathrm{m}$ thickness flat tip.

\section{References}

[1] R. Kodama et al., Nature 412, 798 (2001)

[2] R. Kodama et al., Nature 418, 933 (2002)

[3] H. Azechi et al., Journal of Physics: Conference Series 112, 012002 (2008)

[4] H. Shiraga et al., IFSA 2011 proceedings

[5] S. D. Baton, et al., Phys. Plasmas 15, 042706 (2008)

[6] H.-B. Cai, et al., Physical Review Letters 102, 245001(2009)

[7] T. Johzaki, et al., Nuclear Fusion 51, 073022 (2011) 


\section{IFSA 2011}

[8] A. Sunahara, et al., Laser and particle beams 30, 95 (2012)

[9] K. Nishihara et al., Phys. Plasmas 15, 056708 (2008)

[10] A. Sunahara, et al., Journal of Physics: Conference Series 112, 042048 (2008)

[11] T. Johzaki et al., J. Phys: Conf. Ser. 244, 022040 (2010)

[12] L. Spitzer, Physics of Fully Ionized Gases (Interscience, New York, 1962)

[13] F. Perez et al., Phys. Rev. Lett. 107, 065004 (2011)

[14] T. Johzaki et al., IFSA 2011 proceedings 